\title{
PREDICTING THE BIODEGRADABILITY NATURE OF IMIDAZOLE AND ITS DERIVATIVES BY MODULATING TWO HISTIDINE DEGRADATION ENZYMES (UROCANASE AND FORMIMINOGLUTAMASE) ACTIVITIES
}

\author{
VIJAYAKUMAR VEERARAGAVAN ${ }^{1}$, RADHAKRISHNAN NARAYANASWAMY2*, RAMESHKUMAR CHIDAMBARAM ${ }^{1}$ \\ ${ }^{1}$ Department of Chemistry, Organic Chemistry Laboratory, Veltech Dr. RR and Dr. SR University, Avadi, Chennai, Tamil Nadu, India. \\ ${ }^{2}$ Bio Waste Management Laboratory, Vel Tech Technology Incubator, Veltech Dr. RR and Dr. SR, University, Avadi, Chennai, Tamil Nadu, \\ India. Email: nrkishnan@gmail.com
}

Received: 28 June 2017, Revised and Accepted: 26 July 2017

ABSTRACT

Objectives: The biodegradation pathway of substituted imidazole ring compounds has been reported to have close analogy to the histidine degradation pathway. This prompted the present study to be carried out on 12 selected imidazole and its derivatives which are 1-imidazole, 1, 2-dimethylimidazole, 1-ethyl imidazole, 2-ethyl-4-methylimidazole, 2-isopropylimidazole, 2-Isopropyl-4-nitro-1H-imidazole, 1-methylimidazole, 2-methyl-5-nitroimidazole, 2-methyl-1-vinylimidazole, 1-nitro imidazole, 1-phenyl imidazole, and 1-vinylimidazole.

Methods: The imidazole and its derivatives were evaluated on the docking behavior of urocanase and formiminoglutamase using PatchDock. In addition, molecular physicochemical, drug-likeness, absorption, distribution, metabolism, and excretion analyses (ADME) were done.

Results: The molecular physicochemical analysis revealed that all the tested ligands showed nil violation and complied well with the Lipinski's rule of five. ADME analysis showed that 1-phenylimidazole alone predicated to have cytochrome P450 1A2 inhibition effect. Docking studies revealed that 1-nitroimidazole showed the least atomic contact energy with both targeted enzymes (urocanase and FIGase).

Conclusion: Inhibition of both enzymes (urocanase and FIGase) might show poor biodegradability nature. Thus, we can predict biodegradability nature of imidazloe and its derivatives by modulating two histidine degradation enzymes activities.

Keywords: Biodegradation, Urocanase, Formiminoglutamase, Histidine degradation pathway, Imidazole derivatives.

(C) 2017 The Authors. Published by Innovare Academic Sciences Pvt Ltd. This is an open access article under the CC BY license (http://creativecommons. org/licenses/by/4. 0/) DOI: http://dx.doi.org/10.22159/ajpcr.2017.v10i11.20999

\section{INTRODUCTION}

Imidazole is one of unique five member heterocyclic, aromatic compound with three carbons and two nitrogen atoms in 1 and 3 positions. Imidazole ring is commonly present in the natural occurring compounds, especially in many alkaloids and histidine (amino acid). Imidazole and its derivatives have been reported to show various biological activities such as analgesic, antiaging, antibacterial, anticancer, anticoagulant, antidiabetic, antidepressant, antifungal, anti-inflammatory, antileishmanial, antimalarial, antitubercular, and antiviral. Apart from their pharmaceutical applications, imidazole and its derivatives too have other industrial applications such as prevention of copper corrosion, fire retardant, photography, and in electronics sectors. Imidazole and its derivatives have exhibited wide range of medicinal properties, which urged the medicinal/organic chemists to synthesis several numbers of novel chemotherapeutic agents [1-3]. Iron, copper, and zinc imidazole derivatives have been reported to show corrosion inhibition [4].

The biodegradation pathway of substituted imidazole ring compounds has been reported to have close analogy to the histidine degradation pathway [5]. MetaCyc database (http://metacyc.org) has highlighted three important histidine catabolism/degradation pathways. Urocanase and formiminoglutamase (FIGase) are two key enzymes plays an important role in the histidine degradation pathway. Urocanase has been reported to present in few bacteria, in the liver of several vertebrates/mammals and in plant, especially in Trifolium repens (white clover). Interestingly, urocanase deficiency in the liver causes mental retardation. Urocanase catalyzes the urocanate to 4-imidazolone-5-propionate (one of important intermediate compound of histidine degradation pathway). Similarly, FIGase is another enzyme belongs to arginase/ureohydrolase superfamily, which catalyzes the last step in histidine degradation pathway I through by hydrolysis the imino group of $\mathrm{N}$-formimino-L-glutamate to form formamide and L-glutamate [6-9]. This prompted the present study to be carried out on 12 selected imidazole and its derivatives which are 1-imidazole, 1, 2-dimethylimidazole, 1-ethyl imidazole, 2-ethyl-4-methylimidazole, 2-isopropylimidazole, 2-isopropyl-4nitro-1H-imidazole, 1-methylimidazole, 2-methyl-5-nitroimidazole, 2-methyl-1-vinylimidazole, 1-nitro imidazole, 1-phenyl imidazole, and 1-vinylimidazole. These imidazole and their derivatives were evaluated on the docking behavior of urocanase and FIGase using PatchDock whereby the results have given useful information to predict the biodegradable potential of imidazole and its derivatives. In addition, molecular physicochemical, drug-likeness, absorption, distribution, metabolism, and excretion analyses (ADME) were done.

\section{METHODS}

\section{Ligand preparation}

Chemical structures of the ligands, namely, (a) 1-imidazole (CID 795); (b) 1, 2-dimethylimidazole (CID 15617); (c) 1-ethyl imidazole (CID 81540); (d) 2-ethyl-4-methylimidazole (CID 70262); (e) 2-isopropylimidazole (CID 123457); (f) 2-isopropyl-4-nitro-1H-imidazole (CID 83389); (g) 1-methylimidazole (CID 1390); (h) 2-methyl-5-nitroimidazole (CID 12760); (i) 2-methyl-1-vinylimidazole (CID 76109); (j) 1-nitro imidazole (CID 18208); (k) 1-phenylimidazole (CID 81595); and (l) 1-vinylimidazole (CID 66171) were retrieved from PubMed (www. pubmed.com). The ligands were drawn in ChemBioDraw Ultra 12.0 (www.cambridgesoft.com) and then molecular mechanics (MM2) minimization of ligands were carried out using ChemBio3D Ultra 12.0. Thus, these energy minimized ligands (structures) were employed for PatchDock. 
Target protein identification and preparation

The three dimensional structure of the Pseudomonas putida urocanase (program database [PDB] ID: 1UWL with resolution of $1.76 \AA$ ) and Vibrio cholera FIGase (PDB ID: 1 XFK with resolution of $1.8 \AA$ ) were obtained from the research collaborator for structural bioinformatics protein data bank (anonymous, www.rcsb.org). A chain of both proteins were pre-processed separately by deleting other chains, ligand, as well as the crystallographically observed water molecules (water without hydrogen bonds). Both proteins were prepared using UCSF Chimera software (www.cgi.ucsf.edu/chimera) and resultant proteins were employed for PatchDock.

\section{Molecular descriptors calculation}

Molinspiration online database was used for all selected ligands to calculate thirteen descriptors (www.molinspiration.com) which are log $\mathrm{P}$, polar surface area, molecular weight, number of atoms, number of $\mathrm{O}$ or $\mathrm{N}$, number of $\mathrm{OH}$ or $\mathrm{NH}$, number of rotatable bond, drug-likeness including $\mathrm{G}$ protein coupled receptors ligand, ion channel modulator, kinase inhibitor, nuclear receptor ligand, and number of violations to Lipinski's rule [10].

\section{ADME analysis}

ADME analysis was performed using SwissADME (www.swissadme. ch) online tool and ADME analysis was carried using standard default protocol [11].

\section{Docking studies}

Docking studies were carried out using the PatchDock online server (http://bioinfo3d.cs.tau.ac.il/PatchDock). PatchDock adopts geometrybased molecular docking algorithm method and used to recognize the binding scores, binding residues, and atomic contact energy (ACE) of given ligands [12]. The docking results were obtained through the user email address. We also use to get uniform resource locator which provides the top 20 solutions in a table form through email. From these, top one solution (the docked protein-ligand complex) was selected and downloaded in PDB file format. Further, the binding site analyses were carried using PyMOL software (www.pymol.org).

\section{RESULTS AND DISCUSSION}

In general, biodegradation means the process in which environmental fate of a chemical can be assessed, in other words; it is the ability of any potent microorganisms to transform/use the harmful/hazards chemicals that enters the environment into non-hazards/less hazards one. However, determining the biodegradation nature of compounds under in vitro condition is tedious and time-consuming one [5]. Thus, to overcome problem, molecular docking approaches has been explored in recent years. For instance, molecular modeling of laccase was carried out to study the biodegradation nature of herbicide diuron [13].

The biodegradation pathway of substituted imidazole ring compounds has been reported to have close analogy to the histidine degradation pathway and cyanoimidazole. phenylimidazole and nitro-substitutes imidazoles have been reported to have poor biodegradability nature [5]. The histidine degradation has been reported in several microorganisms including Bacillus subtilis, Enterobacter aerogenes, Pseudomonas aeruginosa, and Pseudomonas fluorescens [14]. Thus, in the present study, imidazole and their derivatives were evaluated on the docking behavior of $P$. putida urocanase and $V$. cholera FIGase using PatchDock.

It is better to know the molecular physicochemical and drug-likeness properties of imidazole and its derivatives, before performing PatchDock. Thus, in the present study all ligands showed nil violation and complied well with the Lipinski's rule of five as shown in Table 1.

In the present study, 1-phenylimidazole exhibited active score $(>0)$ toward ion channel modulator descriptor and interesting none of ligands showing inactive score $(<-5.0)$ toward drug-likeness properties, as shown in the Table 2 .

Table 3 shows the ADME profile, where all the twelve ligands are predicated to have good gastrointestinal (GI) absorption effect, and 1-phenylimidazole alone predicated to have cytochrome P450 1A2 inhibition effect.

Urocanate (first metabolite in the histidine degradation pathway) is converted into 4-imidazolone-5-propionate by the enzymatic action of urocanase. Urocanase has been reported to present in bacteria, vertebrates/mammals, and in plant [7]. The docking studies and binding site analyses in Table 4, shows that 2-ethyl-4-methylimidazole with the highest (ACE [-111.21 kcal/mol]) while 1-nitro imidazole gave the least ACE $(-26.84 \mathrm{kcal} / \mathrm{mol})$ with that of $P$. putida urocanase. The present finding was in good agreement with earlier report, where 2-ethyl-4-methylimidazole exhibited minimum degradation and 1-nitro imidazole shown maximum degradation [5]. These results might suggest that 2-ethyl-4-methylimidazole strongly inhibits urocanase and thus leads to minimum degradation, whereas in case of 1-nitro imidazole it is vice versa. In the present study, interaction with Asp443 amino acid residue has been shown by four ligands, namely, 1-ethylimidazole, 2-methyl-5-nitroimidazole, 1-nitroimidazole, and 1-vinylimidazole. Previously Kessler and coworkers (2004) reported that Asp443 amino acid residue involve in acid-base catalytic reaction, whereas Tyr52 amino acid residue stabilize the positive charge of the substrate [7].

Similarly, the docking studies and binding site analyses in Table 5 , shows that 2-ethylimidazole with the highest ACE $(-32.74 \mathrm{kcal} / \mathrm{mol})$ while 1-nitro imidazole gave the least ACE $(+14.64 \mathrm{kcal} / \mathrm{mol})$ with that of $V$. cholera FIGase. The present finding was in good agreement with earlier report, where 2-ethylimidazole exhibited minimum degradation and 1-nitro imidazole shown maximum degradation [5]. These results might suggest that 2-ethyl-4-methylimidazole strongly inhibits FIGase activity and thus leads to minimum degradation. In case of 1-nitro imidazole

Table 1: Molecular physicochemical descriptors analysis of imidazole and its derivatives using molinspiration online software tool

\begin{tabular}{|c|c|c|c|c|c|c|c|c|}
\hline Ligand & $\log A^{a}$ & TPSA $^{\mathbf{b}}$ & Natoms $^{c}$ & $\mathbf{M W}^{\mathrm{d}}$ & $\mathrm{nON}^{\mathrm{e}}$ & $\mathrm{nOH} \mathrm{NH}{ }^{\mathrm{f}}$ & Nviolations $^{g}$ & Nrotb $^{h}$ \\
\hline 1-imidazole & -0.07 & 28.68 & 5 & 68.08 & 2 & 1 & 0 & 0 \\
\hline 1, 2-dimethylimidazole & 0.08 & 17.83 & 7 & 96.13 & 2 & 0 & 0 & 0 \\
\hline 1-ethyl imidazole & 0.37 & 17.83 & 7 & 96.83 & 2 & 0 & 0 & 1 \\
\hline 2-ethyl-4-methylimidazole & 0.81 & 28.68 & 8 & 110.16 & 2 & 1 & 0 & 1 \\
\hline 2-isopropylimidazole & 1.07 & 28.68 & 8 & 110.16 & 2 & 1 & 0 & 1 \\
\hline 2-Isopropyl-4-nitro-1H-imidazole & 1.15 & 74.51 & 11 & 155.16 & 5 & 1 & 0 & 2 \\
\hline 1-methylimidazole & -0.01 & 17.83 & 6 & 82.11 & 2 & 0 & 0 & 0 \\
\hline 2-methyl-1-vinylimidazole & 0.39 & 17.83 & 8 & 108.14 & 2 & 0 & 0 & 1 \\
\hline 1-nitro imidazole & 0.01 & 74.51 & 8 & 113.08 & 5 & 1 & 0 & 1 \\
\hline 1-phenyl imidazole & 1.27 & 17.83 & 11 & 144.18 & 2 & 0 & 0 & 1 \\
\hline 1-vinylimidazole & 0.30 & 17.83 & 7 & 94.12 & 2 & 0 & 0 & 1 \\
\hline
\end{tabular}

${ }^{\mathrm{a}}$ Octanol-water partition coefficient, ${ }^{\mathrm{b}}$ polar surface area, ${ }^{\mathrm{c}}$ number of non hydrogen atoms, ${ }^{\mathrm{d}}$ molecular weight, ${ }^{\mathrm{e}}$ number of hydrogen bond acceptors ( $\mathrm{O}$ and $\mathrm{N}$ atoms),

${ }^{\mathrm{f}}$ number of hydrogen bond donors (OH and $\mathrm{NH}$ groups), ${ }^{\mathrm{g}}$ number of rule of 5 violations, ${ }^{\mathrm{h}}$ number of rotatable bonds 
Table 2: Drug-likeness property analysis of imidazole and its derivatives using Molinspiration online software tool

\begin{tabular}{|c|c|c|c|c|c|c|}
\hline Ligand & $\begin{array}{l}\text { GPCR* } \\
\text { ligand }\end{array}$ & $\begin{array}{l}\text { Ion channel } \\
\text { modulator }\end{array}$ & $\begin{array}{l}\text { Kinase } \\
\text { inhibitor }\end{array}$ & $\begin{array}{l}\text { Nuclear receptor } \\
\text { ligand }\end{array}$ & $\begin{array}{l}\text { Protease } \\
\text { inhibitor }\end{array}$ & $\begin{array}{l}\text { Enzyme } \\
\text { inhibitor }\end{array}$ \\
\hline 1-imidazole & -3.34 & -3.30 & -3.10 & -3.99 & -3.59 & -2.93 \\
\hline 1, 2-dimethylimidazole & -3.42 & -3.27 & -3.34 & -3.76 & -3.64 & -3.12 \\
\hline 1-ethyl imidazole & -3.50 & -3.46 & -3.09 & -3.76 & -3.65 & -2.68 \\
\hline 2-ethyl-4-methylimidazole & -2.40 & -2.55 & -2.83 & -3.68 & -3.17 & -2.35 \\
\hline 2-isopropylimidazole & -2.57 & -2.22 & -2.68 & -3.58 & -3.11 & -2.40 \\
\hline 2-isopropyl-4-nitro-1H-imidazole & -0.97 & -0.54 & -1.09 & -1.95 & -1.80 & -0.49 \\
\hline 2-methyl-5-nitroimidazole & -2.38 & -1.85 & -2.44 & -3.55 & -3.35 & -1.77 \\
\hline 2-methyl-1-vinylimidazole & -2.97 & -2.86 & -2.62 & -3.70 & -3.54 & -2.22 \\
\hline 1-nitro imidazole & -3.36 & -2.83 & -3.12 & -3.83 & -3.71 & -2.53 \\
\hline 1-phenyl imidazole & -0.72 & 0.07 & -0.58 & -1.60 & -1.04 & -0.19 \\
\hline 1-vinylimidazole & -3.44 & -3.48 & -2.55 & -3.77 & -3.68 & -2.19 \\
\hline
\end{tabular}

*GPCR: G-protein coupled receptors

Table 3: ADME analysis of imidazole and its derivatives using Swiss ADME online tool

\begin{tabular}{|c|c|c|c|c|c|c|c|c|c|}
\hline Ligand & $\mathbf{G l}^{\bullet}$ & BBB" & P-gp & CYP1A2* & CYP2C19* & CYP2C9* & CYP2D6* & CYP3A4* & $\log K p \diamond$ \\
\hline 1-imidazole & High & No & No & No & No & No & No & No & -6.67 \\
\hline 1, 2-dimethylimidazole & High & No & No & No & No & No & No & No & -6.60 \\
\hline 1-ethyl imidazole & High & Yes & No & No & No & No & No & No & -6.25 \\
\hline 2-ethyl-4-methylimidazole & High & Yes & No & No & No & No & No & No & -6.06 \\
\hline 2-isopropylimidazole & High & Yes & No & No & No & No & No & No & -6.11 \\
\hline 2-isopropyl-4-nitro-1H-imidazole & High & No & No & No & No & No & No & No & -6.26 \\
\hline 1 Methylimidazole & High & No & No & No & No & No & No & No & -6.84 \\
\hline 2-methyl-1-vinylimidazole & High & Yes & No & No & No & No & No & No & -6.25 \\
\hline 1-nitro imidazole & High & No & No & No & No & No & No & No & -6.83 \\
\hline 1-phenyl imidazole & High & Yes & No & Yes & No & No & No & No & -5.96 \\
\hline 1-vinylimidazole & High & Yes & No & No & No & No & No & No & -6.50 \\
\hline
\end{tabular}

•GI: Gastrointestinal absorption, "BBB: Blood brain barrier permeant, P-gp: P-glycoprotein substrate, *Inhibitor, ${ }^{\circ}$ skin permeation (cm/s), CYP1: Cytochrome P450 1

Table 4: Binding site analyses of imidazole and its derivatives with that of urocanase using PatchDock

\begin{tabular}{|c|c|c|c|}
\hline Ligand & -ACE (-kcal/mol) & Interaction of amino acid residue & Bond distance ( $($ ) \\
\hline 1-imidazole & 99.75 & No interaction & Nil \\
\hline 1, 2-dimethylimidazole & 31.49 & No interaction & Nil \\
\hline 1-ethylimidazole & 34.87 & Asp443 & 3.50 \\
\hline 2-ethyl-4-methylimidazole & 111.21 & Ser266 & 3.33 \\
\hline 2-isopropylimidazole & 45.48 & Gly324 & 3.34 \\
\hline \multirow[t]{2}{*}{ 2-isopropyl-4-nitro-1H-imidazole } & 55.02 & Thr265 & 3.34 \\
\hline & & Gly324 & 2.99 \\
\hline 1 Methylimidazole & 58.11 & No interaction & Nil \\
\hline 2-methyl-5-nitroimidazole & 40.82 & Asp443 & 3.06 \\
\hline 2-methyl-1-vinylimidazole & 43.91 & Arg455 & 3.46 \\
\hline \multirow[t]{2}{*}{ 1-nitroimidazole } & 26.84 & Tyr52 & 3.25 and 3.35 \\
\hline & & Asp443 & 2.78 \\
\hline 1-phenylimidazole & 79.10 & No interaction & Nil \\
\hline 1-vinylimidazole & 38.28 & Asp443 & 3.04 \\
\hline
\end{tabular}

ACE: Atomic contact energy

positive ACE has been observed, which might be due to unfavorable interaction phenomenon as reported by castro and coworkers [15]. In the present study, interaction with Asp256 amino acid residue has been shown by four ligands, namely, 1, 2-dimethylimidazole, 2-ethyl-4methylimidazole, 2-Isopropylimidazole, and 2-methyl-5-nitroimidazole. Apart from the above, amino acid residues such as Asn59, Asp161, Ser176, Asp254, and Glu300 have been shown to interact with ligands (Imidazole and its derivatives). V. cholera FIGase does not contain bound metal ions, whereas $B$. subtilis FIGase has a binuclear calcium cluster. However, it is not clear that FIGases are calcium ion dependent enzymes or whether they are metalloenzymes at all [16].

\section{CONCLUSION}

In the present study, all the tested ligands have shown to dock with both targeted enzymes. However, 1-phenylimidazole does not interact with any amino acid residues of both targeted enzymes (urocanase and FIGase). Interestingly, among the twelve ligands, 1-nitroimidazole showed the least ACE with both targeted enzymes. Inhibition of both enzymes (urocanase and FIGase) might show poor biodegradability nature. Thus, we can predict biodegradability nature of imidazloe and its derivatives by modulating two histidine degradation enzymes activities. 
Table 5: Binding site analyses of imidazole and its derivatives with that of FIGase using PatchDock

\begin{tabular}{|c|c|c|c|}
\hline Ligand & -ACE (-kcal/mol) & Interaction of amino acid residue & Bond distance $(\AA)$ \\
\hline 1-imidazole & 2.24 & Ser176 & 2.88 \\
\hline 1, 2-dimethylimidazole & 3.46 & Asp256 & 3.17 \\
\hline \multirow[t]{2}{*}{ 1-ethylimidazole } & 32.74 & Ser176 & 3.04 \\
\hline & & Glu300 & 3.33 \\
\hline 2-ethyl-4-methylimidazole & 15.26 & Asp256 & 2.80 \\
\hline 2-isopropylimidazole & 8.93 & Asp256 & 3.45 \\
\hline 1 Methylimidazole & 15.90 & Ser267 & 3.15 \\
\hline \multirow[t]{2}{*}{ 2-methyl-5-nitroimidazole } & 27.63 & Ser176 & 3.55 \\
\hline & & Asp256 & 3.13 \\
\hline \multirow[t]{2}{*}{ 2-methyl-1-vinylimidazole } & 1.18 & Asn59 & 3.51 \\
\hline & & Asp161 & 3.16 and 3.30 \\
\hline \multirow[t]{3}{*}{ 1-nitroimidazole } & +14.64 & Asp161 & $3.13,3.21$ and 3.46 \\
\hline & & Ser176 & $2.75,2.82$ \\
\hline & & Asp254 & and 3.24 \\
\hline 1-phenylimidazole & 30.28 & No interaction & Nil \\
\hline 1-vinylimidazole & 1.25 & No interaction & Nil \\
\hline
\end{tabular}

ACE: Atomic contact energy, FIGase: Formiminoglutamase

\section{ACKNOWLEDGMENT}

The authors express their sincere gratitude to the authority of Veltech Dr. RR and Dr. SR University, Chennai for providing research facilities.

\section{REFERENCES}

1. Shalini K, Sharma PK, Kumar N. Imidazole and its biological activities: A review. Chem Sin 2010;1(3):36-47.

2. Bhatnagar A, Sharma PK, Kumar N. A review on "Imidazoles": Their chemistry and pharmacological potentials. Int $\mathrm{J}$ Pharm Tech Res 2011;3(1):268-82

3. El-Aal EA, Fattah HA, Osman N, Seliem I. Synthesis of novel imidazole and fused imidazole derivatives as cytotoxic and antimicrobial agents: Molecular docking and biological evaluation. Int J Pharm Sci 2015;7(10):36-45.

4. Bhat SU, Naikoo RA, Mir MA, Tomar R. Synthesis of tetra-substituted imidazole derivatives by condensation reaction using zeolite H-ZSM 22 as a heterogeneous solid acid catalyst. Int J Curr Pharm Res 2016;8(1):36-9.

5. Rorije E, Germa F, Philipp B, Schink B, Beimborn DB. Prediction of biodegradability from structure: Imidazoles. SAR QSAR Environ Res 2002;13(1):199-204.

6. Kaminskas E, Kimhi Y, Magasanik B. Urocanase and N-formimino-Lglutamate formiminohydrolase of Bacillus subtilis, two enzymes of the histidine degradation pathway J Biol Chem 1970;245(14):3536-44.

7. Kessler D, Rétey J, Schulz GE. Structure and action of urocanase. J Mol Biol 2004;342:183-94.
8. Tabor H, Mehler AH, Hayaishi O, White J. Urocanic acid as an intermediate in the enzymatic conversion of histidine to glutamic and formic acids. J Biol Chem 1952;196(1):121-8.

9. Yoshida T, Tada K, Honda Y, Arakawa T. Urocanic aciduria: A defect in the urocanase activity in the liver of a mentally retarded. Tohoku J Exp Med 1971;104(4):305-12.

10. Radhakrishnan N, Wai LK, Ismail IS. Molecular docking analysis of natural compounds as human neutrophil elastase (HNE) inhibitors. J Chem Pharm Res 2013;5(10):337-46.

11. Daina A, Michielin O, Zoete V. Swiss ADME: A free web tool to evaluate pharmacokinetics, drug-likeness and medicinal chemistry friendliness of small molecules. Sci Rep 2017;7:42717.

12. Schneidman-Duhovny D, Inbar Y, Nussinov R, Wolfson HJ. PatchDock and SymmDock: Servers for rigid and symmetric docking. Nucleic Acids Res 2005;33:W363-7.

13. Vieira AC, Marschalk C, Biavatti DC, Lorscheider CA, Peralta RM, Seixas FA. Modeling based structural insights into biodegradation of the herbicide diuron by laccase-1 from Ceriporiopsis subvermispora. Bioinformation 2015;11(5):224-8.

14. Bender RA. Regulation of the histidine utilization (hut) system in bacteria. Microbiol Mol Biol Rev 2012;76(3):565-84.

15. Castro JS, Trzaskowski B, Deymier PA, Bucay J, Adamowicz L, Hoying JB. Binding affinity of fluorochromes and fluorescent proteins to Taxol ${ }^{\mathrm{TM}}$ crystals. Mater Sci Eng C 2009;29(5):1609-15.

16. Hai Y, Dugery RJ, Healy D, Christianson DW. Formiminoglutamase from Trypanosoma cruzi is an arginase-like manganese metalloenzyme. Biochemistry 2013;52(51):9294-309. 